\title{
TRANSPARENCIA Y RENDICIÓN DE CUENTAS EN SALUD MATERNA: EL CASO DEL AFASPE EN CHIAPAS
}

María Graciela Freyermuth Enciso ${ }^{1}$ gracielafreyermuth54@hotmail.com

Héctor Javier Sánchez Pérez ${ }^{2}$

hsanchez@ecosur.mx

Hilda E. Argüello Avendaño ${ }^{3}$ hildaeu@gmail.com

\section{RESUMEN}

La Secretaría de Salud (SSA), a través de la Subsecretaría de Prevención y Promoción de la Salud (SPPS), realiza transferencias monetarias y de insumos a las entidades federativas a través del Acuerdo para el Fortalecimiento de las Acciones de Salud Pública en los Estados (AFASPE). En este artículo se describe y analiza el desempeño del AFASPE 2012 en el Programa de Acción Específico Arranque Parejo en la Vida en Chiapas, a la luz del proceso de descentralización. La discusión se centra en el análisis de la planificación, entrega y ejercicio de los recursos, modificaciones del presupuesto, transparencia y rendición de cuentas, que involucran tanto a la SSA como los Servicios Estatales de salud (SESA) y a la Secretaría de Hacienda estatal.

Palabras clave: monitoreo de política pública, transparencia y rendición de cuentas, AFASPE, salud materna.

\section{TRANSPARENCY AND ACCOUNTABILITY IN MATERNAL CARE: THE CASE OF AFASPE IN CHIAPAS}

\begin{abstract}
The Mexican Health Department (Secretaría de Salud / SSA) through the Health Prevention and Promotion SubDepartment (Subsecretaría de Prevención y Promoción de la Salud / SPPS) conducts monetary and input transfers to the states through the Agreement for the Strengthening of Public Health Actions in the States (Acuerdo para el Fortalecimiento de las Acciones de Salud Pública en los Estados / AFASPE). This article describes and analyzes AFASPE's performance in 2012 in the Equal Start in Life Specific Action Program (Programa de Acción Específico Arranque Parejo en la Vida) in Chiapas in the light of the decentralization process. The discussion is focused on the planning analysis, resource delivery and execution, budget modifications, transparency and accountability involving both the SSA and the State Heath Services (Servicios Estatales de Salud / SESA), as well as the Department of Finances of Chiapas State.
\end{abstract}

Key words: public policy monitoring, transparency and accountability, AFASPE, maternal health.

\footnotetext{
${ }^{1}$ Investigadora del Centro de Investigaciones y Estudios Superiores en Antropología Social-Sureste (CIESAS) y secretaria técnica del Observatorio de Mortalidad Materna en México (OMM).

${ }^{2}$ Investigador del El Colegio de la Frontera Sur (ECOSUR) y secretario técnico del OMM Chiapas.

${ }^{3}$ Asistente del Secretariado Técnico del OMM e investigadora asociada, estudiante de doctorado de la Universitat Rovira i Virgili.
} 


\section{INTRODUCCIÓN}

Este artículo describe y analiza el desempeño de los procesos de planificación, gestión, ejecución y transparencia del Acuerdo para el Fortalecimiento de las Acciones de Salud Pública en los Estados (AFASPE), tomando como eje de análisis el caso del presupuesto destinado al Programa de Acción Específico (PAE), Arranque Parejo en la Vida (APV) ahora Programa de Acción Salud Materna y Perinatal. El AFASPE es un mecanismo para transferir recursos financieros e insumos, desde la Subsecretaría de Prevención y Promoción de la Salud (SPPS) de la Secretaría de Salud (SSA) a los Servicios Estatales de Salud (SESA), destinados a los $32 \mathrm{PAE}^{4}$ a través de la firma de un acuerdo marco.

A partir de la descentralización de los servicios de salud en el país, los recursos para el funcionamiento de los SESA se han canalizado a través del Fondo de Aportaciones para los Servicios de Salud (FASSA) del Ramo 33. De acuerdo con la Auditoría Superior de la Federación (ASF) se observa que, en 2010, $83 \%$ de los recursos recibidos en las entidades federativas se ejerció para el pago de nómina, $16.7 \%$ se utilizó para gastos de naturaleza corriente y $0.3 \%$ para erogaciones de inversión (ASF 2010:7).

Aunque el fondo de aportaciones es uno de los presupuestos más importantes para el funcionamiento de los SESA, la federación, a través de la SPPS de la SSA, realiza transferencias monetarias y de insumos para el fortalecimiento de los PAE; sin embargo, hasta 2008 esta transferencia de recursos implicaba una carga administrativa enorme ya que, en palabras de la propia SPPS, cada unidad administrativa y órgano desconcentrado suscribía convenios específicos con las entidades federativas: uno para la transferencia de recursos presupuestarios y otro para la transferencia de insumos, por lo que se firmaban cerca de 400 convenios al año (sólo convenios principales) más

\footnotetext{
${ }^{4}$ Los PAE son los instrumentos rectores en diversos problemas salud, que se elaboran de manera consensuada, basados en las mejores prácticas y en la evidencia científica disponible, con el objetivo de plantear estrategias para la solución de problemas de salud nacionales, así como definir la dirección que todas las instituciones y niveles de gobierno deberán seguir para avanzar de manera conjunta y armónica en los principales retos que enfrenta el Sistema Nacional de Salud. En el caso particular de la salud materna, se dio seguimiento al Convenio AFASPE en el PAE Arranque Parejo en la Vida (APV) (SSA 2008:7).
}

otra serie de convenios modificatorios (SSA, SPPS 2012a:2).

En 2008 se elabora el AFASPE y se desarrolla una aplicación informática: Sistema de Información para la Administración del Fondo de Aportaciones para el Fortalecimiento de Acciones de Salud Pública en las Entidades Federativas (SIAFFASPE), a fin de propiciar la eficiencia, eficacia y transparencia en el manejo de recursos presupuestales para el cumplimiento de metas y objetivos institucionales (SSA, SPPS 2012a:3).

El diseño de un modelo de convenio específico unifica la presentación de información y permite una mejora en la rendición de cuentas. Se semiautomatiza la generación de los convenios específicos y sus correspondientes convenios modificatorios, lo que facilita los trámites administrativos (SSA, SPPS 2012a:3).

El Observatorio de Mortalidad Materna (OMM) se interesó en dar seguimiento a este nuevo modelo de gestión por resultados, que promueve la transparencia y rendición de cuentas. En este trabajo se describen y analizan los procesos de planificación, gestión y ejecución del AFASPE del PAE APV en Chiapas, a la luz del proceso de descentralización del sector salud.

\section{LA DESCENTRALIZACIÓN Y LOS PRESUPUESTOS EN SALUD}

Los procesos de descentralización de los servicios de salud se han dado mediante un marco político-jurídico enfocado en una nueva definición de Estado. El presidente López Portillo adopta, en 1981, una serie de medidas en materia de salud que sentarían las bases para esta nueva reforma. Es con Miguel de la Madrid Hurtado (1982-1988) que inicia el proceso de redefinición del Estado y de un nuevo federalismo. En 1983 y 1984 se publican los decretos que establecen los fundamentos para la descentralización de la Secretaría de Educación Pública, Secretaría de la Reforma Agraria y Secretaría de Salubridad y Asistencia (DOF 1983, 1984). Por tanto, se sientan las bases de la rectoría del Estado en materia de salud y se generan las primeras iniciativas de ley.

La SSA formalizó convenios con los gobiernos de los estados de la República, de acuerdo con el Convenio Único de Desarrollo y a los decretos del 30 de agosto de 1983 y el 8 de marzo de 1984. Estos acuerdos de coordinación tenían un marco legal tan 
amplio, que la descentralización podía realizarse de manera discrecional, dependiendo de la relación entre la SSA y los SESA de cada estado. Los criterios de qué desconcentrar y cómo hacerlo fueron definidos a partir del Ejecutivo federal. Estos convenios se enfocaban a proponer procesos de desconcentración y la SSA seguiría actuando como instancia normativa y de control. Esta primera fase de la descentralización contó con el apoyo de la Presidencia de la República y de la Organización Mundial de la Salud (Soberón y Martínez 1996).

Aunque Cardozo (citado por Torres 1997) señala que en el gobierno de Salinas hubo una amnesia total en cuanto a la descentralización de los servicios de salud, también es cierto que en ese sexenio se realizó el primer convenio con el Banco Mundial, con la finalidad de ampliar la cobertura y fortalecer la infraestructura de las entidades federativas más rezagadas en materia de servicios de salud. En diciembre de 1997 se publica en el Diario Oficial de la Federación (DOF) el Acuerdo de coordinación para la descentralización integral de los servicios de salud en el estado de Chiapas. Este instrumento norma las formas de descentralización, responsabilidades, recursos y decisiones, para fortalecer el nuevo federalismo y acercar a la población servicios básicos, oportunos y eficientes al ser prestados por los estados (DOF 1997). El objetivo de la descentralización es contar con planes y programas que respondan a las necesidades de la población local, que hagan un uso racional y equitativo de los recursos, y que tomen en cuenta las desigualdades y las necesidades de la población.

En este nuevo federalismo se creó el Ramo 33, en el que se encuentran las aportaciones federales para entidades federativas y municipios, que proporcionaría mayores recursos a estados y, al mismo tiempo, procuraría mayor eficiencia y mejores posibilidades de control de los fondos. A diferencia de las participaciones, los fondos de aportaciones son partidas presupuestales etiquetadas ${ }^{5}$ que se ejercen por medio de unidades responsables y municipios. La puesta en marcha del proceso de descentralización coincidió con la transición del partido en el poder y, por tanto, con gobiernos estatales y el de la federación guiados por distintos partidos (2000-2012). La rectoría de la federación se ha visto debilitada frente a los gobier- nos locales que funcionan como entidades autónomas, con mecanismos de transparencia y rendición de cuentas limitados.

Chiapas se vio enormemente favorecido por la descentralización. En 1998 el presupuesto por Ramo 33 Fondo de Aportaciones para los Servicios de Salud (FASSA) fue de $\$ 654961.35$ y el gasto total en salud de \$2 060823.71 (De la Torre 2006). Para el 2012 Chiapas obtuvo por FASSA \$2 909862005.00 (SHCP 2012) y por Seguro Popular (SP) 3533300000.00 (SP 2012) lo que hace un total $\$ 6443162$ 005.00. Es decir, que ha tenido un incremento presupuestal en 3126 veces. Esto sin contar los recursos adicionales que llegan por Ramo 12 a las entidades federativas. Al inicio de la descentralización el Ramo 12 se adelgazó, ya que incluía recursos principalmente para rectoría, y el Ramo 33 para la operación de servicios.

Sin embargo, desde 2003 se inicia un proceso de recentralización en el sector salud que puede reconocerse por el presupuesto destinado a ese rubro mediante los Ramos 12 y 33 . En el cuadro 1 se identifican los montos crecientes de presupuesto a través de los Ramo 33 y Ramo 12: mientras los recursos del Ramo 12 se incrementaron en el periodo casi 22 veces, el Ramo 33 aumentó 13.7 veces. Los recursos del SP tuvieron una tasa media de crecimiento anual (TMCA) de casi 50 veces, y se destinan a los servicios estatales de salud, pero su control lo tiene la federación, a diferencia del FASSA.

La rectoría de la SSA, desde la década de los ochenta, se ha delineado mediante las normas oficiales mexicanas. Estas proporcionan los lineamientos que debe seguir el sector para la atención de los problemas de salud nacionales y, de esta manera, asegurar la alineación de los programas. Otro instrumento son los programas específicos transexenales o sexenales alineados a los planes nacionales de gobierno, en los cuales los titulares del poder Ejecutivo en turno colocan los problemas prioritarios en los que se centrarán sus respectivos gobiernos. Durante 2007-2012 se publicaron los PAE elaborados por las direcciones a cargo de la SPPS.

En este documento se incluyen los resultados del seguimiento de la aplicación del AFASPE para el Programa de Arranque Parejo en la Vida (materna perinatal) en el estado de Chiapas.

\footnotetext{
${ }^{5}$ Etiquetar los presupuestos significa destinarlos explícitamente a un sector de la población o a una actividad en particular.
} 
Cuadro 1. Estructura del presupuesto por fuente de financiamiento 2003-2011 (millones de pesos).

\begin{tabular}{|c|c|c|c|c|c|c|c|c|c|c|}
\hline Ramo & 2003 & 2004 & 2005 & 2006 & 2007 & 2008 & 2009 & $\begin{array}{l}\text { PEF } \\
2010\end{array}$ & $\begin{array}{l}\text { PEF } \\
2011\end{array}$ & TМСА \\
\hline $\begin{array}{l}\text { Total } \\
\text { Ramo } 12\end{array}$ & 17720.4 & 23694.0 & 33705.5 & 41547.7 & 53144.0 & 69743.5 & 80989.1 & 89892.9 & 105313.9 & 21.9 \\
\hline $\begin{array}{l}\text { Nivel } \\
\text { federal }\end{array}$ & 16070.2 & 19003.1 & 24975.4 & 24849.4 & 27200.7 & 33492.9 & 37426.9 & 36048.6 & 42956.0 & 11.5 \\
\hline $\begin{array}{l}\text { Seguro } \\
\text { Popular }\end{array}$ & 1650.2 & 4690.9 & 8730.1 & 16698.3 & 25943.3 & 36250.6 & 43562.2 & 53844.3 & 62357.9 & 49.7 \\
\hline Ramo 33 & 32936.3 & 34532.1 & 38042.6 & 40674.3 & 44466.2 & 47689.9 & 48617.8 & 53100 & 55698.7 & 6.0 \\
\hline TOTAL & 50656.7 & 58226.1 & 71748.1 & 82222.0 & 97610.2 & 117433.4 & 129606.9 & 142992.9 & 161012.6 & 13.7 \\
\hline
\end{tabular}

Fuente: Dirección General de Programación (2011).

\section{LA MORTALIDAD MATERNA (MM) EN MÉXICO Y CHIAPAS}

De acuerdo con información oficial, en el país la MM ha descendido paulatinamente. Mientras en 1955 la razón de muerte materna (RMM) era de 204.4 defunciones maternas por cada 100000 nacidos vivos, en 1990 era de 89.0; en el año 2000 de 72.6 y en 2006 fue de 60.0 en igual denominador (SSA 2008). La meta establecida para el año 2015 es reducir la RMM a una no mayor de 22 por 100000 nacidos vivos. Siguiendo con las cifras oficiales, en términos absolutos las MM han disminuido de 2203 ocurridas en 1990 en todo el país, a 1166 en 2006 y se espera que, de alcanzar la meta en 2015, cuando mucho ocurran 417 defunciones (SSA 2008). Según el sector salud, la disminución de la MM se explica por la ampliación de la cobertura de atención de parto por personal calificado a partir de la incorporación de municipios indígenas al SP, de $70 \%$ en el año 2000 a $94.1 \%$ en 2006 (SSA 2008:20), y a que se redujo el número de mujeres que fallecieron en el hogar sin haber recibido atención médica, de $17 \%$ a $13.5 \%$, entre 2002 y 2006 (SSA 2008).

En el periodo 2007-2011, la RMM aún está muy lejos de llegar a dicha meta de 2015. En 2007 la RMM fue de 48.1, en 2008 de 49.2, en 2009 de 53.3, en 2010 de 44.1 (Freyermuth y Luna 2010, Freyermuth et al. 2011) y en 2011 de 43.2 con las proyecciones del censo de 2010 (Freyermuth et al. 2012). Chiapas, Guerrero y Oaxaca son los estados con mayor
RMM con cifras por arriba de 60 por 100 mil nacidos vivos (Freyermuth et al. 2013).

Chiapas es uno de los estados con menos de $60 \%$ en la reducción planificada (OMM 2013a), y entre 2009 y 2010 tuvo una disminución de la RMM de alrededor de 5\% (OMM 2013b). Según las numeralias 2010 y 2011 del OMM, para Chiapas: tuvo una disminución de 8 puntos en la RMM de 2010 a 2011 (de 60.2 a 52.6), incrementó el porcentaje de muertes maternas en indígenas de $25 \%$ a $50 \%$ y en beneficiarias del SP de $34 \%$ a $58 \%$.

En 2012 se tuvieron 960 muertes maternas registradas en todo el país, con una $\mathrm{RMM}^{6}$ de 42.7 por 100 mil nacimientos. De esas 960 muertes maternas, 68 (7.1\%) correspondían a Chiapas, por lo que el estado se ubicaba como el tercero con mayor MM del país (sólo detrás del Estado de México y Veracruz). Para ese año, Chiapas tuvo un incremento de 1.3 puntos porcentuales en su RMM con respecto al promedio 2006-2011. ${ }^{7}$

\footnotetext{
${ }^{6}$ En los últimos dos años la DGIS ha cambiado los denominadores, así en el 2011 oficialmente cambiaron las proyecciones de CONAPO 2005-2030 con la actualización de nacidos vivos estimados de las proyecciones de población del CONAPO 20102050 y a fines de 2013 la DGIS normó el cálculo de la RMM con certificados de nacimiento SINAC, esto ha determinado la disminución de por lo menos en 10 puntos en la RMM de casi todas las entidades federativas.

${ }^{7}$ SSA, SPPS, Dirección General de Epidemiología, Dirección General Adjunta de Epidemiología, Sistema Nacional de Vigilancia Epidemiológica (SINAVE), 2012.
} 


\section{MORTALIDAD PERINATAL Y NEONATAL}

En 1980, 67\% de las muertes neonatales eran debidas a afecciones propias del periodo perinatal y a anomalías congénitas, en 2002 ascendió a 95\%. En dicho periodo las muertes neonatales asociadas con diarrea e infección respiratoria disminuyeron de 25 a menos de $1 \%$ (SSA 2008:19).

En 2002, de las 44000 muertes infantiles registradas, $27000(62.5 \%)$ ocurrieron en el periodo neonatal y, de estas, 20000 fueron neonatales tempranas, de las cuales 9000 ocurrieron en las primeras 24 horas posteriores al nacimiento (SSA 2008:18). Entre los años 2000 y 2006 se registró una disminución de $20.8 \%$ en la mortalidad neonatal. De las principales causas de muerte perinatal, la de nacimiento prematuro ocupó el primer lugar, con $43 \%$ del total de causas, seguida de las infecciosas $(23.2 \%)$ y la hipoxia-asfixia (17\%), que históricamente se mantenía en el primer lugar (SSA 2008:22). Para disminuir las posibilidades de defectos al nacimiento y la muerte neonatal se vinculó, a partir del año 2000, una serie de estrategias como la administración de ácido fólico, tamiz neonatal, prevención de infecciones de transmisión sexual (ITS) y reanimación neonatal, entre otras.

\section{ACUERDO PARA EL FORTALECIMIENTOS DE LAS ACCIONES DE SALUD PÚBLICA EN LOS ESTADOS (AFASPE). EL CASO DE CHIAPAS}

El 29 de febrero de 2008, la SSA ${ }^{9}$ y el Ejecutivo del estado de Chiapas celebraron el Acuerdo Marco de Coordinación en Materia de Transferencia de Recursos Federales, con objeto de fijar las bases y los mecanismos generales para la prestación de servicios en materia de salubridad general, con calidad y calidez, de acuerdo con los objetivos y metas señalados en el Programa Sectorial de Salud (PROSESA) 2007-2012.

\footnotetext{
${ }^{8}$ De acuerdo con la Organización Mundial de la Salud (OMS), la mortalidad perinatal es la que ocurre de la semana 22 del embarazo al séptimo día de nacimiento. A su vez, la mortalidad neonatal es la que ocurre entre el nacimiento y el 28 día de vida. La neonatal puede ser temprana o precoz (primeros siete días de vida) y tardía (después del séptimo día, pero antes de los 28 días posteriores al nacimiento) (SSA 2008:18).

${ }^{9}$ Para hacer referencia a la Secretaría de Salud a nivel federal, se utilizará el nombre «La Secretaría» o bien las siglas «SSA».
}

Dichos recursos han sido transferidos mediante el Convenio Específico en Materia de Transferencia de Subsidios para el Fortalecimiento de Acciones en Salud Pública en las entidades federativas. ${ }^{10}$

El convenio marco fue suscrito por parte del estado de Chiapas por el secretario de Salud y director general del Instituto de Salud del Estado de Chiapas (ISECH) y el Secretario de Hacienda (poder Ejecutivo del estado), y a el nivel federal por el subsecretario de SPPS, asistido por los titulares de las unidades administrativas y los órganos desconcentrados que tiene adscritos (la Secretaría). En Chiapas, el Convenio AFASPE correspondiente a 2012 se firmó el 26 de marzo de dicho año.

El Convenio consta de tres apartados (antecedentes, declaraciones y cláusulas) y cuatro anexos (A): A1. Copias fotostáticas de los nombramientos de los titulares de la Secretaría; A2. Identificación de la fuente de financiamiento de los programas; A3. Calendario de las ministraciones; A4. Metas e indicadores de los programas en materia de salud pública y compromisos que asume el poder Ejecutivo del estado y el Ejecutivo federal. Este último puede ser una guía para la evaluación y el control del ejercicio.

En el caso del PAE APV se establecieron las siguientes metas para 2012:

- $\quad 100 \%$ de mujeres embarazadas a las que se les realizan las pruebas de detección para sífilis y $\mathrm{VIH} / \mathrm{sida}$.

- $25 \%$ de personal de salud de los hospitales resolutivos capacitado en emergencias obstétricas.

- $\quad 22 \%$ de personal de salud involucrado con la atención del recién nacido en los hospitales de segundo nivel de atención, capacitado en reanimación neonatal.

- Cuatro cursos de capacitación en lactancia materna realizados contra programados en hospitales

\footnotetext{
${ }^{10}$ En su origen, este instrumento también contemplaba asesoría técnica, supervisión en la aplicación de los recursos, evaluación del cumplimiento de objetivos y metas, rendición de cuentas y transparencia de los recursos. Fue considerado como un mecanismo de alineación presupuestal para que, a través de un solo convenio, se transfirieran recursos a la entidad de diferentes fuentes, y también fue previsto que la compra de los insumos se realizara con efectividad (OMM 2012).
} 
- $100 \%$ de adquisición de ácido fólico para el consumo anual de $25 \%$ de las mujeres en edad fértil responsabilidad de la SSA.

- $\quad 100 \%$ de recién nacidos con tamiz neonatal.

- Realizar $100 \%$ de diagnóstico, tratamiento y seguimiento de los casos con errores innatos del metabolismo.

- Notificación inmediata de $100 \%$ de las defunciones maternas al Sistema de Vigilancia Epidemiológica.
La transferencia de recursos federales presupuestales de la SSA al estado de Chiapas para el PAE APV 2012 fue de $\$ 6823238.03$ pesos (\$4 112690.43 en recursos financieros y $\$ 2710547.60$ en insumos). Esto representó casi $25 \%$ de los recursos asignados a los siete programas que coordina el Centro Nacional de Equidad de Género y Salud Reproductiva (CNEGySR). Los programas de este centro se llevaron $10 \%$ de los recursos asignados a Chiapas y al PAE APV le correspondió $2.6 \%$ (cuadros 2 y 3 ).

Cuadro 2. Monto de recursos federales transferidos por la SSA al poder Ejecutivo estatal para el Convenio AFASPE-Chiapas, 2012, en los relativo al programa Arranque Parejo en la Vida.

Monto de los recursos financieros e insumos del Convenio AFASPE APV Chiapas 2012

Recursos federales que destina la SSA a la entidad en recursos financieros y/o insumos

Total para APV Chiapas

Total para Programas CNEGySR en Chiapas

Total de recursos federales a transferir a Chiapas

$\%$ de APV en relación con el total del CNEGySR

$\%$ de APV en relación con el total federal

$\%$ de CNEGySR con relación al total federal

Monto máximo a cargo de la SSA (pesos)

Recursos financieros

4112690.43

21677897.40

67216255.70

18.97

6.11

32.25
Insumos

2710547.60

5300394.50

195622954.90

51.13

1.39

2.70
Total 6823238.03 26978291.90 262839210.60

25.29

2.59

10.26

Fuente: Elaboración propia a partir de SSA, SPPS (2012b).

Cuadro 3. Monto de recursos federales transferidos por la SSA al poder Ejecutivo del estado para el Convenio AFASPE-Chiapas, 2012, por Programa de Acción Específico a cargo del Centro Nacional de Equidad de Género y Salud Reproductiva.

\section{Monto de los recursos financieros e insumos del Convenio AFASPE APV Chiapas 2012}

\section{Programa}

Monto máximo a cargo de la SSA (pesos)

\section{Cáncer de mama}

Cáncer cérvico-uterino

APV

Planificación familiar y anticoncepción

Salud sexual y reproductiva del adolescente

Prevención y atención de la violencia familiar y de género

Igualdad de género en la salud

Total del CNEGySR

Gran Total de recursos federales a transferir a la entidad

\begin{tabular}{|r|r|r|}
\hline Recursos financieros & \multicolumn{1}{|c|}{ Insumos } & \multicolumn{1}{c|}{ Total } \\
\hline 1228622.00 & 0.00 & 1228622.00 \\
\hline 1676991.43 & 3941786.75 & 5618778.18 \\
\hline 2625000.00 & 2618934.80 & 5243934.80 \\
\hline 0.00 & 2162090.00 & 2162090.00 \\
\hline 1243843.98 & 149839.00 & 1393682.98 \\
\hline 1000000.00 & 0.00 & 1000000.00 \\
\hline 0.00 & 7920.00 & 7920.00 \\
\hline 41648676.78 & 188986727.80 & 230635404.58 \\
\hline
\end{tabular}

Fuente: Elaboración propia a partir de SSA, SPPS (2012b). 
El Convenio establece que los recursos federales transferidos conservan su carácter federal, por lo que su control, verificación, seguimiento y fiscalización se llevará a cabo de conformidad con las disposiciones jurídicas vigentes en materia del ejercicio del gasto público federal, en tanto que la ministración de dichos recursos se hará considerando tanto la estacionalidad del gasto como, en su caso, el calendario presupuestario autorizado por la SHCP, a través del Sistema Integral de Administración Financiera Federal (SIAFF). También se establece que serán radicados en la Secretaría de Hacienda (SH) del estado, en tanto que los insumos serán entregados directamente al ISECH para ser aplicados, de manera exclusiva, en los PAE, entre los que se encuentra el de APV.

Se establece que las transferencias de recursos e insumos no son susceptibles de presupuestarse en los ejercicios fiscales siguientes, ${ }^{11}$ y que el alcance de las metas comprometidas será evaluado de acuerdo con

${ }^{11}$ De acuerdo al convenio, la SSA y el ISECH deberán sujetarse a los siguientes parámetros para asegurar la transparencia en la aplicación y comprobación de los recursos federales transferidos: a) Los recursos financieros e insumos que la Secretaría se compromete a transferir, estarán sujetos a la disponibilidad presupuestaria y a las autorizaciones correspondientes, de conformidad con las disposiciones jurídicas aplicables y de acuerdo con el calendario que para tal efecto se establezca. $b$ ) La Secretaría, por conducto de las unidades administrativas u órganos desconcentrados que tienen a cargo cada uno de los programas, podrá verificar, supervisar y evaluar en todo momento las acciones objeto de este convenio, así como la aplicación y destino de los recursos financieros e insumos federales transferidos. c) La Secretaría, por conducto de las unidades administrativas u órganos desconcentrados que tienen a cargo cada uno de los programas, aplicará las medidas que procedan de acuerdo con la normatividad aplicable, los casos en que los recursos financieros no hayan sido aplicados para los fines objeto del presente convenio, o bien en contravención a sus cláusulas, ocasionando como consecuencia la suspensión o cancelación de la ministración de recursos federales. $d$ ) La Secretaría, por conducto de las unidades administrativas $u$ órganos desconcentrados que tienen a cargo cada uno de los programas, practicará, cuando lo considere necesario, visitas a los programas convenidos a efecto de observar sus avances, estando obligado el poder Ejecutivo del estado a la entrega de certificación del gasto, que sustente y fundamente la aplicación de los recursos.

Dichos recursos, una vez devengados y conforme avance el ejercicio, deberán ser registrados por el poder Ejecutivo del estado en su contabilidad y se rendirán en su cuenta pública, sin que por ello pierdan su carácter federal.

La certificación del gasto deberá reunir los requisitos enunciados en los artículos 29 y 29-A del Código Fiscal de la Federación y, en su caso, la Secretaría solicitará la documentación que ampare la relación de gastos CONVENIO-AFASPE-CHIS/2012, pp. 7-8. los indicadores establecidos en el A4. Se especifica, asimismo, que la aplicación de recursos financieros e insumos transferidos se destinarán de forma exclusiva a intervenciones de los programas.

Los remanentes, fruto de ahorros en el ejercicio de los recursos financieros, previa autorización de las instancias correspondientes, se podrían aplicar en otras intervenciones, o bien a otros o a los mismos conceptos de gastos del mismo Programa que fueran necesarios para el cumplimiento de sus metas, siempre y cuando se ejercieran antes del 31 de diciembre de 2012 y se demostrara que se cumplió totalmente con las metas establecidas. En la sexta cláusula del Convenio se señalan las obligaciones del Ejecutivo estatal; en la octava, los recursos presupuestarios federales que se le transfieren; en la novena, la vigencia del Convenio; en la décima se señala que el Convenio podrá modificarse de común acuerdo y por escrito, sin alterar su estructura y en estricto apego a las disposiciones jurídicas aplicables; y la undécima trata de las causas de terminación del Convenio específico.

\section{METODOLOGÍA}

Durante el segundo semestre del 2012 se dio seguimiento al AFASPE del PAE APV a través del modelo de monitoreo de políticas públicas diseñado exprofeso (Freyermuth y Argüello 2013) por integrantes del OMM. La información fue recabada en dos fases: durante la primera se realizó trabajo de gabinete (documentación) basado en la revisión de la iniciativa. La información se obtuvo al consultar distintas fuentes, la disponible en la página web del OMM (www.omm. org.mx) y la solicitada al ISECH y a la SSA a través del sistema INFOMEX (www.infomex.org), del Instituto Federal de Acceso a la Información (IFAI). ${ }^{12} \mathrm{~A}$ nivel estatal, los datos llegaron con lentitud y fueron insuficientes respecto de los intereses de la investi-

${ }^{12}$ Véase Manual del modelo, en el apartado de anexos se ejemplifica la solictud ante el INFOMEX que es un sistema electrónico creado por el Instituto Federal de Acceso a la Información y Protección de Datos (IFAI) con financiamiento del Banco Mundial, que facilita y mejora los procesos, tareas y avances en la gestión de solicitudes de información; brinda atención a aquellos organismos, entidades y dependencias que se hayan incorporado al sistema, para recibir y contestar eficientemente las solicitudes de información. Los números de solicitud para la certificación del gasto fue 0001200334312 y para los informes trimestrales 0001200334212 , se solicitó lo mismo con la opción de la plataforma a nivel estatal: 5861 y 5862 respectivamente. 
gación. Sin embargo, a nivel federal la información fue suficiente y adecuada.

En la segunda fase se realizaron entrevistas en la SH del estado de Chiapas y en el ISECH. En la SH, se entrevistó, el 6 de diciembre de 2012, a la directora general de Planeación de la Subsecretaría de Planeación, Presupuesto y Egresos y al director de Política del Gasto en sus oficinas de Tuxtla Gutiérrez. La solicitud de entrevistas en la SH se realizó mediante un oficio fechado el 8 de noviembre de 2012, dirigido al entonces secretario de Hacienda del estado de Chiapas, en el que se comunicaba el interés en entrevistar al propio secretario, al subsecretario de Planeación, Presupuesto y Egresos, al director de Política del Gasto y a uno de sus asesores. El tiempo transcurrido entre la entrega de esa solicitud y la realización de las entrevistas fue de prácticamente un mes.

Las entrevistas en el ISECH se pidieron mediante un oficio fechado el 8 de noviembre de 2012, dirigido al entonces secretario de Salud, y tardaron en realizarse un mes y una semana, debido al proceso de entrega-recepción a las nuevas autoridades del ISECH. ${ }^{13}$ Se realizaron el 14 de diciembre de 2012, con el jefe del Departamento de Integración Presupuestal de la Dirección de Planeación y Desarrollo; y ese mismo día tuvo lugar una entrevista grupal con el coordinador administrativo de Salud Pública, la encargada del PAE APV, el excoordinador de Salud Materna y Perinatal, con un médico de apoyo en el Programa de Fuerza y Tarea en Atención Médica y el coordinador de proyectos específicos de la subdirección de Atención Hospitalaria. Respecto de los obstáculos enfrentados en el trabajo de campo, destacan los siguientes:

\section{RESULTADOS}

\section{RECURSOS APORTADOS POR LA FEDERACIÓN AL PAE APV Y CONVENIOS MODIFICATORIOS}

La federación originalmente contempló transferir al ISECH $\$ 2625000.00$ pesos en recursos financieros y $\$ 2618934.80$ en insumos. Sin embargo, en 2012 hubo dos convenios modificatorios realizados por el nivel central. En el primero se benefició la suma destinada a Chiapas (57\% más) y el monto de recursos

\footnotetext{
${ }^{13}$ En 2012 hubo cambio de gobierno estatal en Chiapas. Concluyó la gestión de Juan Sabines Guerrero e inició la de Manuel Velasco Coello.
}

financieron pasó a \$4 112690.43 pesos y en insumos se le asignaron $\$ 91612.80$ pesos más (cuadro 2). El segundo no impactó en el monto presupuestal para este programa. El personal del ISECH mencionó que usualmente los convenios modificatorios son realizados desde el nivel central. También mencionaron que en 2012 uno de los convenios modificatorios incluyó recursos para contratar médicos ginecobstetras pero solo por una quincena, y para compra de insumos, que por su monto requerían licitación. Por ello, explican, parte de estos recursos no se ejercieron.

\section{PLANIFICACIÓN DEL PRESUPUESTO DEL AFASPE}

De acuerdo con la información proporcionada por el excoordinador del PAE APV, hasta el año 2010, el ISECH no participaba en el proceso de planificación: «el estado o la federación aportaba cierta cantidad, de acuerdo a los criterios de evaluación que ellos hacían de cada uno de los programas para los estados y ellos aportaban cierta cantidad y ya venía etiquetado, a dónde iba a ser ejercido ese presupuesto» (CPAPV-CPE-CASP-ECPAPV_14/12/12).

A partir de 2011 hay mayor intervención del ISECH y en 2012 la federación incluye al ISECH sobre sus requerimientos. En enero de 2012 se tuvo una reunión de planificación y se hizo una programación a nivel estatal en la que participaron las áreas de Atención Médica, Salud Reproductiva y administrativa. Entre estas áreas hicieron una proyección de presupuesto de «ciento veintitantos millones de pesos y lo enviamos». Pero de esa programación, el ISECH no recibió lo que proyectó; la federación, de acuerdo con su metodología y criterios (los cuales mencionaron desconocer), otorgaron un presupuesto de alrededor de $\$ 2600000.00$ pesos en recursos económicos y otros dos millones y tanto de insumos. En este sentido cabe señalar que se subejerció gran parte del presupuesto de 2011, lo que probablemente determinó que para 2012 recibieran $27 \%$ de lo destinado en 2011 y, con los convenios modificatorios, se incrementó una la tercera parte de lo asignado para ese año.

El excoordinador del PAE APV hizo hincapié en la notable reducción de la cantidad presupuestada, y que estaba «perfectamente» etiquetado para su ejercicio, lo cual no fue provechoso para el ISECH. Señaló que los criterios de planificación que ellos 
habían utilizado para la solicitud de recursos para el año 2012 fueron epidemiológicos (sitios de mayor ocurrencia de defunciones maternas y de demanda de atención de urgencias maternas) y el de la infraestructura existente (incluyendo el número de personal capacitado). El entrevistado señaló que el proceso de planeación duró más de un mes y varias sesiones, tratando de hacer coincidir las políticas del nivel federal, el OMM y otros organismos, tales como incluir la equidad, el respeto a los cultos de la población y la interculturalidad (por ejemplo, la inclusión de dos mesas para parto vertical) (CPAPV-CPE-CASP-ECPAPV_14/12/12).

Es evidente que los fondos del AFASPE son utilizados fundamentalmente para incluir aquellas acciones que la federación considera estratégicas. Podemos hacer este juicio porque los convenios son muy similares a lo largo de todo el territorio nacional: existen fuertes indicios de que desde el nivel central se decide la planificación, y de que el punto de vista, en este caso del ISECH, es tomado en cuenta de manera marginal. También es cierto, como ya se mostró, que el estado de Chiapas cuenta con recursos propios para poner en marcha sus políticas estratégicas.

\section{FIRMA DEL CONVENIO}

Las personas entrevistadas del ISECH desconocían la fecha exacta de firma del Convenio, acertaron a decir que había sido en el mes de marzo. El proceso de la firma es el siguiente: el nivel federal envía el Convenio para la firma del secretario de Salud (en este caso, el ISECH) y del secretario de Hacienda. Una vez firmado por ambos, se envía a la SPPS y de ahí a todas las direcciones para que se recaben las firmas. En el caso de los entrevistados en la SH, sí conocían la fecha de la firma del Convenio. Este proceso se ha venido agilizando y no representa ningún obstáculo en su operación.

\section{ENTREGA Y EJERCICIO DE RECURSOS}

De acuerdo con la información proporcionada por el coordinador administrativo de Salud Pública del ISECH, el ejercicio de recursos se realiza con apego a lo establecido en el Convenio, e incluso señaló que es uno de sus candados (CPAPV-CPE-CASP-ECPAPV 14/12/12). El director de Política del Gasto de la SH mencionó que él había revisado el Convenio y que no había detectado un calendario específico para realizar las ministraciones al PAE APV 2012, pero que «las ministraciones se están haciendo en función a como quedó plasmado en el Convenio»». Asimismo, desconocía la fecha de recepción de la primera ministración de recursos de AFASPE del PAE APV: «un dato preciso así, no lo tengo presente, porque los oficios de autorización aún están ahorita recabándolos porque son varios, no los tengo ahorita a la mano, pero si gustas, en un rato más me lo suben, $\mathrm{y}$ te doy, con precisión, en qué fecha fue la primera ministración de recursos» (DGP-DPG_6/12/12).

No estaba al tanto si las ministraciones del PAE APV se habían recibido (desde el nivel federal) de acuerdo con lo establecido en el Convenio, así como las fechas de ministraciones al ISECH, y solo señaló que el depósito de recursos desde el nivel federal «normalmente se hace conforme a lo que estipule el Convenio» y que las transferencias al ISECH se hacen «casi de inmediato, máximo cinco o diez días, dado que el Convenio señala que debe asignarse de manera inmediata a la ejecutora, casualmente para atender las necesidades en la materia por la cual fue firmado el Convenio» (DGP-DPG_6/12/12).

Para que la entidad reciba recursos del Convenio y, particularmente a los programas específicos del CNEGySR, la SH señaló que por ser un tema más operativo, el ISECH era el que contaba con todos los elementos al respecto, que la SH, como dependencia normativa, lo que hace es cuidar que se cumpla con el Convenio, que el dinero se destine a lo estipulado, y radicar el recurso para que el ISECH lo ejecute.

El coordinador administrativo de Salud Pública del ISECH manifestó que si bien el uso de la plataforma del SIAFFASPE ${ }^{14}$ facilita la disponibilidad de recursos (es más fácil saber cuándo y para qué programa fueron depositados), también obstaculiza su ejecución,

\footnotetext{
${ }^{14}$ Sistema de Información para la Administración del Fondo para el Fortalecimiento de Acciones en Salud Pública en las Entidades Federativas. Su objetivo general es permitir a la SSA a través de la SPPS, «alinear, controlar y automatizar el uso y ejecución de los recursos presupuestales y programáticos, así como las acciones en salud pública... con el fin de obtener eficiencia financiera y el logro de metas a través de un instrumento de participación, federal y estatal, que asegure los recursos disponibles e impulse el desarrollo de las acciones de salud pública, de acuerdo con su misión institucional». El SIAFFASPE permite identificar metas a cumplir y conocer el destino del gasto, mejorar los mecanismos de colaboración, transferencia, transparencia y rendición de cuentas entre la federación y los estados (cfr. SSA, SPPS s/f).
} 
debido a que en el nivel federal se registran insumo, costo y cantidad, y eso implica que el ISECH se tiene que apegar estrictamente a lo que ahí se estipula, con el gran inconveniente de que los costos que se establecen desde el nivel federal no corresponden a los costos en Chiapas. Cuando disminuyen o se incrementan, tienen que pedir autorización a México para poder comprar. Sólo si en el nivel federal aprueban la compra, entonces la hacen. En caso de no seguir estos procedimientos el nivel federal no les acepta las comprobaciones, con el argumento de que «no está en apego a los costos o cantidades estipulados en el AFASPE» (CPAPV-CPE-CASP-ECPAPV_14/12/12).

El coordinador de Proyectos Específicos de la subdirección de Atención Hospitalaria agregó que, desde el punto de vista técnico, los costos que se etiquetan en el nivel federal son excesivamente inferiores y no se ajustan al costo real del mercado local. Por ejemplo, si el nivel federal etiqueta cierto equipo en $\$ 25000$ pesos, en el mercado dicho equipo que cumpla la norma puede costar entre $\$ 160000$ y $\$ 170000$ pesos. En otras palabras, «el centro hace su programación de acuerdo a los costos que ellos manejan». El nivel central pide acatar los precios programados y ahí es cuando surge la pregunta «¿Somos descentralizados o somos centralizados? La respuesta es que seguimos siendo centralizados porque el centro nos marca las acciones que tenemos que hacer» (CPAPV-CPE-CASP-ECPAPV_14/12/12).

Cuando hay demora en la liberación de recursos por parte del nivel federal, el ISECH se ve afectado porque carece de la certidumbre y liquidez necesarias para realizar la compra de insumos. Así, proveedores que en cierto momento le daban un precio determinado de algún producto, lo incrementan por la demora en el pago. En consecuencia, hay ocasiones en que no han podido comprar las cantidades que tenían previstas e incluso no han podido adquirir determinado producto. Además, tienen la restricción de que no pueden utilizar dinero de otra partida sin la autorización correspondiente, la cual puede ser denegada, «porque, ¿de dónde voy a sacar si me ponen candados de que yo no puedo sacar dinero de otra partida? Usted nada más tiene la partida $X$ para lo que va a comprar y san se acabó, si no lo compra ése es su problema, si le alcanza o no le alcanza» (CPAPV-CPE-CASP-ECPAPV_14/12/12). A decir del entrevistado - con asentimiento del resto de participantes en la entrevista- esta situación afecta prácticamente a $100 \%$ de las compras programadas. Desde la visión del personal del ISECH entrevistado, los dos aspectos que más afectan la disponibilidad de recursos son:

Hasta 2011, Hacienda descontaba el uno al millar de los recursos que venían para el ISECH. A partir del 2012, ya no se les hace dicho descuento.

La entrega de recursos no se realiza en el tiempo estipulado, el proceso que se tiene que seguir para su liberación está normado por la SH: en cuanto «México» envía el recurso a la $\mathrm{SH}$, manda al ISECH, vía el SIAFFASPE, una notificación con la fecha y el monto del depósito. Con eso el ISECH solicita a la SH el recibo de ese dinero y con dicho recibo se inicia el trámite de solicitud de recursos. Para todo recurso de AFASPE se tiene que hacer un expediente en el que se especifique en qué está programado el gasto, de acuerdo con lo que dice la federación (lo que incluye tipo de insumos y montos; el ISECH no puede modificar cantidades, sino sujetarse a lo que dice la federación); dicho expediente debe contener el Convenio ya firmado, ya que si no, no se puede hacer uso de recurso alguno. Para el año 2012, el ISECH llegó a un acuerdo con la SH para que con el borrador del Convenio que se envía para la firma del secretario se pueda iniciar el proceso de liberación de recursos.

El expediente se entrega al área administrativa del ISECH, que se coordina con el representante del ISECH ante Hacienda y hace todos los trámites. Si el expediente entra en la SH antes del día 15 de determinado mes, Hacienda lo procesa y para el día 30 de ese mes libera los recursos o autoriza que ya se puedan cobrar. En el caso de que aún no se hayan recibido ministraciones, se envía el expediente y aunque Hacienda haya autorizado el recurso, hay que esperar a que llegue la ministración y enviar el recibo. En Hacienda este proceso involucra dos áreas diferentes: una que recibe el dinero y notifica, y otra a la que hay que solicitar el dinero. La versión de la SH de este proceso, coincide con la del ISECH. Desde el ISECH, en este proceso también es importante mencionar los siguientes tres aspectos:

- Como requisito de la SPPS, se abren dos cuentas: una por parte de la SH y otra por parte del ISECH.

- La coordinación del PAE APV (al igual que en otros programas) no maneja dinero, este llega al área administrativa del ISECH y sólo le in- 
forman a la Coordinación que el dinero está ahí para destinarse al Programa.

Hasta el año 2010 había muchos problemas para identificar a qué dirección o programa pertenecían los recursos enviados por parte de la federación. Tenían que estar preguntando en varias áreas su procedencia, montos y fechas de depósitos. Con las modificaciones hechas a la plataforma del SIAFFASPE se resolvió de manera significativa este problema.

En el ISECH el ejercicio de recursos es competencia del responsable de la Coordinación Administrativa de Salud Pública. Dicha coordinación se formó por el aumento de recursos en cada uno de los proyectos y por las múltiples actividades que tenía cada coordinador de proyecto o de programa. Anteriormente, cada coordinador hacía todas las gestiones, tales como la de recursos materiales, el área administrativa y tesorería. Ahora el coordinador administrativo funciona como un enlace, al cual cada coordinador le presenta su expediente con su respectiva programación y las necesidades de licitación. En esta coordinación se ve todo lo que son las requisiciones de compras, se verifica la suficiencia presupuestal, se etiqueta el recurso y se efectúan las solicitudes al área correspondiente, por ejemplo, si hay que adquirir recursos materiales se lleva a dicha área, ahí verifican los montos y determinan el proceso de adquisición.

A pregunta expresa sobre quién es el encargado de ejecutar el recurso con respecto a una meta específica, por ejemplo la de capacitación del personal de salud de los hospitales resolutivos en emergencias obstétricas, la encargada del PAE APV señala que el recurso lo ejecutó el área de Enseñanza de Educación en Salud del ISECH a nivel estatal y que no solo se utilizó el recurso del AFASPE, sino también recursos propios del área de Educación en Salud para poder lograr la meta. Los entrevistados hacen notar que en el ISECH, a partir de 2012, se está intentando realizar las actividades del AFASPE en coordinación y con apoyo de otras áreas del ISECH, como en el ejemplo de este caso, el área de Enseñanza. Otro ejemplo mencionado fue la detección del VIH en embarazadas, la cual se trabaja de manera conjunta con el SP: para el AFASPE no se dieron pruebas de ELISA, pero en el SP sí se contempla dicho insumo, así como también pruebas para sífilis.
A partir de lo anteriormente expuesto, podemos identificar las siguientes problemáticas:

- Falta de flexibilidad por parte de la federación para hacer ajustes a los costos de insumos;

- La federación no especifica las características de los equipos e insumos que tienen en su catálogo de precios;

- Falta de claridad de los criterios de asignación de recursos del nivel federal al estatal;

- El proceso de entrega de recursos por parte de la SH es tardado,

- Notificación tardía en el caso de que existan convenios modificatorios.

\section{MODIFICACIONES AL CONVENIO}

Tal como ya se mencionó, en la firma del Convenio se establece que puede ser sujeto de modificaciones de común acuerdo y por escrito.

En el primer convenio modificatorio al AFASPE (SPPS 2012a), suscrito el 29 de junio de 2012, como se mencionó al inicio de la exposición de resultados, se incrementó la totalidad de los recursos en $57 \%$ para un total de $\$ 6823238.03$. En dicho convenio modificatorio se estableció como calendario de ministraciones de recursos el mes de mayo (\$2 625 000.00) y junio (\$1 487690.43). ${ }^{15}$ No hubo cambio en las metas establecidas. ${ }^{16}$ En el segundo convenio modificatorio (SPPS 2012b), suscrito el 28 de septiembre de 2012, no se afectó el monto de recursos a transferir ni de insumos, ni las fechas de ministración de recursos y las metas establecidas, salvo la relativa a «Porcentaje de notificación inmediata de las defunciones maternas al Sistema de Vigilancia Epidemiológica», que en el Convenio original y en el primer convenio modificatorio estaba en $100 \%$ y en este segundo convenio modificatorio se establece en $60 \%$.

De acuerdo con la información obtenida en el ISECH, las principales modificaciones a los montos de los recursos transferidos del nivel federal al estatal se realizan precisamente en esa dirección, es decir, de la federación al estado de Chiapas, en tanto que en el ISECH las únicas modificaciones que llegan a realizar, previa solicitud de autorización para ello,

\footnotetext{
${ }^{15}$ Anexo 3 del Primer convenio modificatorio, p. 9.

${ }^{16}$ Anexo 4 del Primer convenio modificatorio, pp. 18-19.
} 
son para comprar insumos que llegan a diferir del precio presupuestado (bien sea a la alza o a la baja) en el nivel federal, o bien para la transferencias de recursos de una partida a otra.

\section{COMPROBACIÓN}

El Convenio estipula las fechas de las comprobaciones. Primero se envían los comprobantes escaneados, el nivel federal coteja y, si da el visto bueno a la comprobación, se envía la documentación original. La coordinación administrativa de Salud Pública conjunta la documentación y la entrega al área administrativa del ISECH y de ahí se envía al CNEGySR. En esta instancia los representantes entregan la comprobación, que es analizada por los responsables federales. Las comprobaciones tienen que coincidir con lo que está en la plataforma SIAFFASPE (cantidades y precios).

\section{TRANSPARENCIA Y RENDICIÓN DE CUENTAS}

De acuerdo con la información obtenida en el ISECH, el responsable de este aspecto es el área jurídica, encargada, entre otras acciones, de publicar el Convenio en la página web del ISECH. Por otra parte, para las solicitudes de transparencia hay un área de apoyo y seguimiento, donde se reciben las solicitudes y las dirigen a la dirección que corresponda, si es de AFASPE, a la Dirección de Salud Pública y ya ahí deciden si contesta el director o la turna a la subdirección correspondiente. El área de apoyo y seguimiento es la encargada de hacer llegar de forma oficial la información solicitada.

En la entrevista realizada en la $\mathrm{SH}$, el director de Política del Gasto señaló que ellos, tanto a nivel estatal como federal, no tienen la facultad de fiscalizar-auditar la ejecución de recursos del AFASPE, por lo que sólo dan seguimiento del cumplimiento de metas y gastos. Por otra parte, sobre la publicación de resultados del ejercicio de recursos del AFASPE a través de la cuenta pública, el citado funcionario señaló que una vez que la cuenta pública es aprobada por el Congreso, se convierte en documento oficial y se publica en la página de Hacienda y que ese es un medio oficial de rendición de cuentas, pero que también estaba en otros sistemas, como el sistema de formato único que se instituyó desde 2009: «Todos los reportes trimestrales y de cierres, ahí están, los datos específicos, del Convenio en mención»
(DGP-DPG_6/12/12). Sin embargo, el Convenio no es publicado en la página web del ISECH, ni se puede acceder directamente a los informes trimestrales o la certificación del gasto.

\section{SEGUIMIENTO DEL CUMPLIMIENTO DE METAS DEL AFASPE 2011}

Los aspectos comprometidos en el Convenio 2011 fueron: $100 \%$ de hospitales resolutivos con plantillas completas, en sus tres turnos, con insumos necesarios para la atención de las emergencias obstétricas y equipamiento para atenderlas; realización de pruebas de detección para sífilis y $\mathrm{VIH} /$ sida a $85 \%$ de mujeres embarazadas; realización de $90 \%$ de las pruebas de tamiz neonatal; $80 \%$ en diagnóstico, tratamiento y seguimiento a casos de errores innatos del metabolismo; $100 \%$ de notificación inmediata de defunciones maternas en el Sistema de Vigilancia Epidemiológica; $50 \%$ de visitas para evaluación de la capacitación de parteras tradicionales; adquisición de $100 \%$ de ácido fólico para el consumo anual de $25 \%$ de las mujeres en edad fértil; $80 \%$ de compromisos cumplidos, adquiridos por el Comité Estatal de Mortalidad Materna y Perinatal.

El presupuesto total de 2011 para el PAE APV fue de $\$ 19412754.12$ pesos (financiero $\$ 15237109.00$ pesos y $\$ 4175645.12$ pesos en insumos). Para pago de nómina se gastaron $\$ 2987066.00$ pesos en contrataciones en tres hospitales, en uno básico comunitario y en cuatro centros de salud. Estos cuatro últimos no están alineados con las metas propuestas. En equipo e instrumental médico se gastaron $\$ 6709515.50$ pesos; $\$ 800000.00$ pesos en combustible y \$2 122899.94 pesos en medicamentos (cabe señalar que no se especifica el tipo de medicamentos) y $\$ 54000$ pesos en dispositivos intrauterinos que no corresponden a este Programa; $\$ 30359.98$ pesos para tiras reactivas, $\$ 120000.00$ pesos sin descripción y $\$ 43410.00$ pesos para mobiliario, que hacen un total de $\$ 12872251.60$ pesos. Como puede observarse, en 2011 no se gastó el dinero que se les asignó para ese año y hubo gastos que no estuvieron dirigidos al cumplimiento de las metas del PAE APV.

\section{CONCLUSIONES}

El Convenio AFASPE 2012 del PAE APV es un instrumento de transferencia de recursos financieros e 
insumos a los estados a fin de definir metas estratégicas por parte de la federación. Si tomamos en cuenta que en 2012 Chiapas recibió alrededor de 6 400 millones de pesos y por AFASPE 262 millones, la mayor parte de estos en insumos (195 millones), se puede reconocer que no es una parte sustantiva de su presupuesto, sino una estrategia para el fortalecimiento de ciertas acciones. Sin embargo, los sistemas de evaluación y seguimiento a los que el AFASPE está sujeto favorecen la transparencia y la rendición de cuentas. En ello radica su importancia.

Según la perspectiva del ISECH todavía existen dificultades para el cumplimiento de los AFASPE, entre las que destacan:

Que no se toman en cuenta las necesidades del estado de Chiapas en el proceso de planificación, el ISECH desconoce los criterios del nivel central para integrar la planificación del presupuesto en el AFASPE y la asignación es menor a lo que solicitan. No obstante, en años previos el ISECH no gastó los recursos asignados.

Sobre la entrega de recursos, se obtuvo información contradictoria: por una parte, el coordinador administrativo de Salud Pública refirió que se hacía con apego a lo establecido y que de hecho ese aspecto era uno de los «candados» del Convenio y, por otra, personal operativo manifestó que el recurso llegaba muy tarde (entre agosto y septiembre), etiquetado y rígido para cantidades y montos de insumos.

Los dos convenios modificatorios del 2012, realizados por el nivel central, beneficiaron con mayores recursos (57\% más). Esto evidencia una programación deficiente por parte de la federación. Posiblemente estos convenios son utilizados para redistribuir recursos que no son utilizados por el CNEGySR, y el subejercicio recae en las entidades federativas y no en la federación.

La plataforma SIAFFASPE, implementada en 2010, representa ventajas y desventajas para el personal del ISECH. El dinero que se transfiere del nivel central es fácilmente ubicable en términos de montos, fechas y programa al que va dirigido. Entre las principales desventajas se encuentran la falta de flexibilidad entre los costos del catálogo de la federación en relación con los costos locales de los insumos o equipos, lo que dificulta el ejercicio del gasto.

Hasta el año 2011, la SH retenía el uno al millar de los recursos que eran transferidos desde el nivel central. Desde este año ya no se da tal situación.
No se encontró, ni se obtuvo, informe trimestral alguno por parte del ISECH.

El ISECH no tiene publicado el Convenio AFASPE 2012 en su página web.

\section{RECOMENDACIONES SOBRE EL AFASPE PAE APV}

A partir de los resultados del monitoreo, el OMM emite las siguientes recomendaciones: $a$ ) Fortalecer la transparencia y rendición de cuentas en Chiapas, publicando la distribución y la certificación del gasto. b) Fortalecer la contraloría social de las organizaciones de la sociedad civil incluyéndolas desde la redacción del Convenio, a fin de agilizar los procesos de vigilancia de la ejecución de recursos. c) Adecuar la coordinación entre los niveles central y estatal, a través de una planificación conjunta. d) Disminuir el número de convenios modificatorios y agilizar de esta manera la transferencia de los recursos. e) Definir claramente el catálogo de costos a partir de la especificación de insumos, equipos y servicios a adquirir. $f$ ) Que el CNEGySR solicite la entrega puntual de informes trimestrales y la certificación del gasto que sustenten la aplicación de recursos, y que revise el apego a lo programado en el SIAFFASPE y que la SH cumpla con su obligación de validar el resultado de las evaluaciones hechas a los SESA.

\section{FUENTES DE CONSULTA}

Auditoría Superior de la Federación (ASF), 2010, «Auditorías al Fondo de Aportaciones para los Servicios de Salud (FASSA)», Cámara de Diputados, México, en www.ofsnayarit.gob.mx/ capacitacion/2010/0615_1m01.pdf [consulta: 1 de diciembre de 2012].

Diario Oficial de la Federación (DOF), 1983, «Decreto por el que el Ejecutivo Federal establece bases para el programa de descentralización de los servicios de salud de la Secretaría de Salubridad y Asistencia», martes 30 de agosto, Secretaría de Gobernación, México, en dof.gob.mx/ nota_to_imagen_fs.php?cod_diario $=207621 \&$ pagina $=11 \&$ seccion $=0$ [consulta: 10 de diciembre del 2012].

1984, «Acuerdo por el que se desconcentran las farmacias de interés social de la Secretaría de Salubridad y Asistencia», jueves 30 de 
agosto, Secretaría de Gobernación, México, en dof.gob.mx/nota to imagen_fs.php?codnota $=4686120 \&$ fecha $=30 / 08 / 1984 \&$ cod_diario $=201915$ [consulta: 10 de diciembre del 2012].

1997, «Acuerdo de Coordinación para la descentralización integral de los servicios de salud en el Estado de Chiapas», martes 16 de diciembre, Secretaría de Gobernación, México, en dof.gob.mx/nota_to_imagen_fs.php?codnota $=4903917 \&$ fecha $=16 / 12 / 1997 \&$ cod_diario $=209812$ [consulta:10 de diciembre del 2012].

Dirección General de Programación, 2011, «Proceso de integración programática presupuestal 2012, FASSA del Ramo 33», 8 de julio, Secretaría de Salud, México, en web.salud.gob.mx:8080/ appdgpop/IPP_2012/1FCH.pdf [consulta: 12 de diciembre de 2013].

Freyermuth, Graciela, Marisol Luna, 2010, Numeralia 2009. Mortalidad Materna en México, CIESAS/Fondo de Población de las Naciones Unidas (UNFPA)/Organización Panamericana de la Salud (OPS)/Observatorio de Mortalidad Materna en México (OMM), en www.omm.org. $\mathrm{mx} /$ images/stories/Documentos\%20grandes/Numeralia $\% 202009 \% 20$ isbn.pdf [consulta: 21 de diciembre de 2012].

Freyermuth, Graciela, Marisol Luna, Pilar Ochoa, 2011, Numeralia 2010. Mortalidad Materna en México, CIESAS/OPS/OMM, en www.omm.org.mx/ images/stories/Documentos\%20grandes/Numeralia\%202010\%20nueva.pdf [consulta: 21 de diciembre de 2012].

Freyermuth, Graciela (coord.), Marisol Luna, Hilda E. Argüello, 2012, Muerte Materna. Indicadores 2010, CIESAS/OPS/IPAS México/OMM, México, en www.omm.org.mx/ images/stories/Documentos\%20grandes/I2010B.pdf [consulta: 14 de enero de 2013].

Freyermuth, Graciela, Marisol Luna, José Alberto Muños, 2013, Numeralia 2011, Mortalidad Materna en México, CIESAS/Fundación MacArthur en México/ OMM en México, México, en www.omm.org.mx/images/stories/Documentos\%20 grandes/ DG\%20Numeralia\%202011\%20ÚLTIMO\%20corregida\%20.pdf [Consulta: 9 de octubre de 2013].
Freyermuth, Graciela, Hilda E. Argüello, 2013, Manual Operativo. Modelo de monitoreo de políticas públicas, CIESAS/Fondo de Población de las Naciones Unidas (UNFPA), México, en omm.org.mx/images/stories/Documentos $\% 20$ grandes/pdf MMPP\%20MANUAL\%20OPERATIVO\%20FINAL\%20enero,\%202014.pdf [consulta: 25 de enero de 2013].

Observatorio de Mortalidad Materna en México (OMM), 2012, Monitoreo AFASPE 2011, Observatorio de Mortalidad Materna en México, México, en omm.org.mx/index.php/monitoreo-de-politicas-publicas/167.html [consulta: 4 de junio 2012].

, 2013a, Porcentaje de Avance por entidad federativa en el Objetivo 5 de Desarrollo del Milenio entre 1990 y 2011, México, en www.omm. org.mx/images/stories/Documentos\%20grandes/ ODM_10.jpg [consulta: 20 diciembre de 2013]. , 2013b, Disminución de la RMM de 2010 a 2011 de acuerdo con los compromisos de los SESA, México, en www.omm.org.mx/images/stories/ Documentos\%20grandes/sesa_10.jpg [consulta: 20 diciembre de 2013].

Secretaría de Hacienda y Crédito Público (SHCP), 2012, Presupuesto de Egresos de la Federación para el ejercicio fiscal 2012, Gobierno de México, en: www.apartados.hacienda.gob.mx/presupuesto/temas/pef/2012/ [consulta: el 10 de julio 2013].

Secretaría de Salud (SSA), 2008, Programa de Acción Especifico 2007-2012 Arranque Parejo en la Vida, Gobierno de México, en www.spps. gob.mx/arranque-parejo-en-la-vida [consulta: 20 de diciembre 2012].

Sistema Nacional de Vigilancia Epidemiológica (SINAVE), 2012, Informe Semanal de Vigilancia Epidemiológica. Defunciones Maternas, Informe epidemiológico hasta la semana 52, Dirección General de Epidemiología, Secretaría de Salud, en www.omm.org.mx/images/ stories/Documentos\%20grandes/52\%202012. pdf [consulta: 11 de febrero de 2013].

Secretaría de Salud (SSA), Subsecretaría de Prevención y Promoción de la Salud (SPPS), 2012a, Acuerdo para el Fortalecimiento de Acciones de Salud Pública en los Estados, presentación de 45 diapositivas en Power Point proporcio- 
nada en sesión de trabajo con la Dirección de Operación de la SPPS, 2 de marzo 2012.

—, 2012b, Convenio AFASPE-Chis/12, en www. spps.gob.mx/images/stories/SPPS/Docs/convenios/ modificatorio1_afaspe_2012/01-CM-AFASPECHIS-2012.pdf [consulta: 14 febrero 2013].

—, s/f, Sistema de Información para la Administración del Fondo para el Fortalecimiento de Acciones de Salud Pública (SIAFFASPE), en www.spps.gob.mx/avisos/1232-siaffaspe.html [consulta: 27 de mayo de 2013].

Subsecretaría de Prevención y Promoción de la Salud (SPPS), 2012a, Primer convenio modificatorio-AFASPE 2012, México, en www.spps. gob.mx/images/stories/SPPS/Docs/convenios/modificatorio1_afaspe_2012/01-CM-AFASPECHIS-2012.pdf [consulta: 1 de marzo, 2013].

- 2012b, Segundo convenio modificatorio-AFAS$P E$ 2012, México, en www.spps.gob.mx/images/ stories/SPPS/Docs/convenios/modificatorio2 afaspe_2012/02-CM-AFASPE-CHIS-2012.pdf [consulta: 1 de marzo, 2013].

Soberón, Guillermo y Gregorio Martínez, 1996, «La Descentralización de los Servicios de Salud en México en la década de los ochenta», Salud Pública, v. 38, n. 5, México, en bvs.insp.mx/ rsp/articulos/articulo.php?id=000967\#bibliografia [consulta: 7 de junio 2013]

Torre, Cecilia de la, 2006, «El Proceso de Descentralización y los Presupuestos del Sector Salud en el Estado de Chiapas: un análisis del Fondo de Aportaciones para los Servicios de Salud y su impacto en la muerte materna», tesis de licenciatura, Universidad Autónoma de Chiapas, México.

Torres, Antonio, 1997, Descentralización en Salud: algunas consideraciones para el caso de México, Documento de Trabajo 69, CIDE, División de Administración Pública, México.

\section{ENTREVISTAS}

Clave: DGP-DPG_6/12/12 Mtra. María del Pilar Cariño Sarabia, directora general de Planeación (DGP), Subsecretaría de Planeación, Presupuesto y Egresos, y Lic. Odilio Pérez Vicente, director de Política del Gasto (DPG), Secretaría de Hacienda y Crédito Público, 6 de diciembre de 2012.

Clave: CPAPV-CPE-CASP-ECPAPV_14/12/12

Dra. Leticia Raquel Ramírez Alfaro, coordinadora del Programa APV (CPAPV); Ernesto Polens Guillén, Programa de Fuerza y Tarea, en Atención Médica; Javier Ordoñez Rodríguez, Coordinación de Proyectos Específicos de la Subdirección de Atención Hospitalaria (CPE); Ricardo Alejandro Alvarado Jiménez, coordinador administrativo de Salud Pública (CASP); Dr. José de Jesús Romero Ángeles, excoordinador estatal de Salud Materna y Perinatal (ECPAPV), Instituto de Salud del Estado de Chiapas, 14 de diciembre de 2012
Fecha de recepción: 5 de septiembre de 2013.

Fecha de aceptación: 25 de noviembre de 2013. 